\title{
À la rencontre de l'Autre au Moyen Âge, In memoriam Jacques Le Goff
}

Laurence Moal

\section{(2) OpenEdition}

1 Journals

Édition électronique

URL : https://journals.openedition.org/abpo/4213

DOI : $10.4000 /$ abpo.4213

ISBN : 978-2-7535-7720-6

ISSN : 2108-6443

Éditeur

Presses universitaires de Rennes

Édition imprimée

Date de publication : 18 décembre 2018

Pagination : 178-180

ISBN : 978-2-7535-7718-3

ISSN : 0399-0826

\section{Référence électronique}

Laurence Moal, « À la rencontre de l'Autre au Moyen Âge, In memoriam Jacques Le Goff », Annales de Bretagne et des Pays de l'Ouest [En ligne], 125-4 | 2018, mis en ligne le 18 décembre 2018, consulté le 28 février 2022. URL : http://journals.openedition.org/abpo/4213; DOI : https://doi.org/10.4000/abpo. 4213 
"polygonale ", préfigurant longtemps à l'avance les forts détachés des frontières de l'Est des débuts de la III République.

La fin du XVII ${ }^{\mathrm{e}}$ siècle vit la modification du logis de François II, peut-être (mais ce n'est pas certain), à la suite d'un incendie, aboutissant à l'édification du Grand Gouvernement, prolongé par l'aile du Lieutenant du roi, détruite le 25 mai 1800 par la terrible explosion de la poudrière abritée dans la tour des Espagnols; outre l'anéantissement de la chapelle ducale (où eurent lieu le 8 janvier 1499 les espousailles d'Anne et de Louis XII) et de la salle des archives, elle fit 60 morts et 108 blessés, dont tous les noms sont rappelés de façon touchante à notre mémoire. En 1814, le château n'était plus " considéré comme un poste fortifié " par la Commission du classement des places de guerre; tout au plus servit-il de dépôt de bouches à feu et de leurs boulets, comme le montre une étonnante photographie prise en 1858, année d'un projet de restauration (inabouti) de la tour du Fer à Cheval. En 1862 le château fut inscrit sur la liste des monuments historiques, mais dès 1854 les façades du Grand Logis avaient fait l'objet d'importantes restaurations, préludes à de longues campagnes " gothicisant " la place forte, la dernière achevée en 2007. Ces travaux firent d'ailleurs l'objet de vifs débats entre archéologues du bâti, tenants de restitutions à l'identique, et architectes, interprétant « la restauration comme un acte architectural, donc créateur ", querelles multiséculaires qui ne seront sans doute jamais tranchées. Toujours est-il que l'impression d'entrer dans un " château neuf " est flagrante; la patine du tufeau s'atténuera au fil du temps, permettant des photographies un peu moins surexposées (en raison du trop beau temps!) que plusieurs de celles offertes dans ce, au demeurant, magnifique ouvrage.

Tout semble avoir été dit, et de belle façon, sur le château. Tout? L'histoire n'est heureusement jamais terminée et de nouveaux matériaux peuvent servir à prolonger son discours. Ainsi, a-t-il été très récemment porté à notre connaissance trente-neuf dessins réalisés en Loire-Inférieure entre les années 1834 et 1839 par le commissaire de Marine Félix Marant-Boissauveur, dont quatre représentent le château de Nantes (un en 1834, trois en 1836) [DELouche, Denise, Guigon, Philippe (dir.), avec les contributions d'Alain Boulaire, Anna CoRKhill, René Estienne et Yann Guesdon, Félix Marant-Boissauveur (1821-1900). Album breton, Rennes, Presses universitaires de Rennes, 2017] : même produits par un artiste amateur, ils apportent des informations inédites sur cette icône de la ville, et au-delà, de la province.

Philippe GUIGON

Josserand, Philippe et PySIAK, Jerzy (dir.), À la rencontre de l'Autre au Moyen Âge, In memoriam Jacques Le Goff. Actes des premières Assises franco-polonaises d'histoire médiévale, Rennes, PUR, 2017, 246 p., $23 €$.

Les premières Assises franco-polonaises d'histoire, rassemblées en juin 2014, marquent la volonté de célébrer la mémoire de Jacques Le Goff, décédé quelques semaines plus tôt, et de renouer avec une entreprise qu'il avait initiée dans les années 1950. L'éminent médiéviste découvre la Pologne en 1958, alors qu'il est envoyé en mission par Fernand Braudel dans le cadre d'un échange avec l'institut d'histoire de l'Académie polonaise. Les nombreux séjours qu'il y effectue par la suite pour raisons familiales ou scientifiques, témoignent à la fois de son inclination à aller à la rencontre de l'Autre, de son engagement dans la lutte pour les droits de l'homme et de ses convictions européennes. Avec ce volume publié aux Presses universitaires de Rennes, les auteurs, sous la direction de Philippe Josserand et 
Jerzy Pysiak, ont voulu souligner les liens étroits qu'entretenait Jacques Le Goff avec ce pays tout en rendant hommage à l'un de ses champs de recherche privilégiés : l'Autre et l'altérité au Moyen Âge. Dix médiévistes, cinq pour la France, autant pour la Pologne, apportent leur contribution à ce volume avec des " perspectives temporelles, thématiques, géographiques et culturelles variées ". On ne peut que saluer cette initiative de mieux saisir la figure de l'Autre, cet « inconnu sur lequel on projette ses préjugés " (Jean-Claude Schmitt, p. 17), alors que la " crise migratoire " actuelle éveille chez certains Européens des craintes propices à la diffusion de nouveaux stéréotypes et des réactions qui vont à l'encontre des valeurs que prétend défendre l'Europe.

Après un hommage à Jacques Le Goff, Jean-Claude Schmitt retrace soixante ans de collaboration et d'amitié entre médiévistes polonais et français, une coopération entre l'Académie des sciences et la section de l'EPHE (puis l'EHESS). La contribution d'Henryk Samsonowicz étudie la notion d'altérité en Europe centrale et orientale du haut Moyen Âge, en s'intéressant aux motivations qui conduisaient les peuples à entamer des contacts avec leurs voisins, proches ou lointains : la nécessité d'acquérir des biens matériels par le biais des échanges commerciaux, les guerres entre chefferies puis les premiers États, la soif de découverte. Les noms de peuples vivant sur les terres du nord et de l'est de l'Europe énumérés par les savants de l'Antiquité tardive, puis par les auteurs de l'époque ultérieure, témoignent de ces rencontres et de l'émergence du concept d'identité. Mateusz Wilk envisage l'évolution de la littérature apocalyptique latine dans la péninsule ibérique de l'Antiquité tardive jusqu'à la seconde moitié du $\mathrm{IX}^{\mathrm{e}}$ siècle, en centrant son analyse sur le rôle de l'islam dans ces sources. Annick Peters-Custot s'interroge sur ce qu'est être "grec " dans les sources latines de l'Italie du VIII ${ }^{\mathrm{e}}$ au XI ${ }^{\mathrm{e}}$ siècle, et en particulier sur l'usage du terme de Grecus pour qualifier ce qui relève du monde byzantin. Une partie du corpus étudié est constituée de textes polémiques, écrits dans un contexte de tensions entre l'Occident, la papauté et l'Empire d'Orient, en particulier au moment de la question des successeurs de la Rome antique et par celle de la primauté pontificale. Le discours s'appuie sur une vision très négative des Grecs, façonnée par des topoi et stéréotypes. En revanche, hors des polémiques impériales et pontificales, dans un contexte littéraire apaisé, les auteurs latins reconnaissent parfois la légitimité du monopole de l'Empire d'Orient sur le qualificatif de "romain " voire d'empereur. Cependant, malgré l'importance de la spécificité juridique et le particularisme liturgique des Byzantins pour les auteurs latins, la conscience de l'unité et de l'identité dans le christianisme n'est pas remise en cause.

L'article suivant porte sur « la ville, espace d'expérience de l'Autre : les marqueurs de l'altérité à Cordoue, des Omeyyades aux Almohades (VIII'-XIII ${ }^{\mathrm{e}}$ siècle) ". S'appuyant sur des œuvres littéraires arabes et les archives du sol, Christine Mazzoli-Guintard analyse différentes facettes de l'altérité dans un cadre urbain exceptionnel de l'histoire d'al-Andalus : représentations, pratiques topographiques, religieuses et linguistiques, ainsi que ses gradations, de l'acceptation au rejet de l'étranger. Elle s'intéresse notamment aux espaces de l'expérience de l'Autre féminin. La différence suscite une mise en altérité très codifiée visant à empêcher la mixité et à réguler les contacts entre Cordouans et Cordouanes. Roman Michałowski s'interroge, quant à lui, sur ce qui fait la différence entre le paganisme et le christianisme aux yeux d'auteurs carolingiens et ottoniens, comme Ermold le Noir, Raoul et Méginhard de Fulda puis Brunon de Querfurt. Les païens, aux yeux des chrétiens, sont des "Autres ». Ils sont accusés de cupidité, de cruauté, de bestialité et d'adorer les idoles. Mais il faut nuancer selon les sources. L'altérité ressentie n'est pas aussi forte dans tous les cas et elle ne signifie pas toujours étrangeté. Pour Ermold le Noir, par exemple, les Danois n'ont 
jamais été tout à fait étrangers, car c'était d'eux que les Francs tiraient leur origine. De la même manière, les barbares constituent un anti-modèle aux yeux des Byzantins. Mais cette opposition est trop réductrice, comme le constate Nicolas Drocourt dans son étude de la correspondance officielle des basileis avec leurs voisins bulgares au début du $\mathrm{x}^{\mathrm{e}}$ siècle, qui lui permet de nuancer cette opposition entre Byzantins et barbares. Deux figures émergent dans ces sources épistolaires : les Sarrasins et les Perses, des voisins à part à la périphérie de l'Empire, parfois même proposés comme des modèles lorsque les circonstances l'exigent. Philippe Josserand centre, lui, son analyse sur les relations à l'Autre, parfois inattendues, qui se nouent entre les frères des ordres militaires et les Infidèles à la frontière de la chrétienté latine. À partir de la fin du Moyen Âge, les querelles entre chrétiens conduisent à la valorisation du prophète de l'islam. John Tolan examine les écrits de plusieurs auteurs, tel Michel Servet ou Henry Stubbe et montre que Mahomet devient un modèle pour les réformateurs religieux et politiques au XVII ${ }^{\mathrm{e}}$ siècle. S'appuyant sur des relations des premières expéditions portugaises (Xve-début du XVI ${ }^{\mathrm{e}}$ siècle), Michał Tymowski s'intéresse à la diversité des peuples et des cultures africaines observée et décrite par les Européens. Comme les Portugais ont besoin de partenaires commerciaux, ils vont à la recherche d'informations sur l'organisation des différents peuples côtiers. L'expérience de la découverte puis de la connaissance de l'Autre pose la question de la différence entre Africains et Européens, mais aussi de la différenciation entre différents groupes d'Africains. Pour certains auteurs, cela conduit à repenser la conception de l'homme et à percevoir l'humanité dans sa diversité et sa complexité. Pour d'autres, cette diversité devrait céder la place à l'unité par l'adoption du baptême. Enfin, Patrick Boucheron clôt ce volume avec un article intitulé " Le Moyen Âge à l'épreuve du monde : entre altérité et familiarité ».

Laurence MOAL

Leloup, Daniel, Rennes une capitale en pan-de-bois, Skol Vreizh, coll. « Demeures remarquables de Bretagne ", Morlaix, 2017, 136 p.

Malgré les destructions importantes liées à l'incendie de 1720 ou à celles plus récentes de la période contemporaine, Rennes reste la cité bretonne la mieux dotée en immeubles à pan-de-bois. L'existence d'un ouvrage entièrement dédié à ce sujet est amplement justifiée, d'autant plus qu'aucune étude, avant celle de Daniel Leloup, ne traitait du pan-de-bois de manière aussi transversale et diachronique. L'ouvrage richement illustré suit un plan chronologique et propose de parcourir plus de 500 ans d'évolution stylistique de ce type d'architecture, en mettant l'accent sur les continuités et les ruptures. Il dépasse l'analyse strictement typo-chronologique en posant une question centrale : quel regard a-t-on posé sur l'architecture à pan-debois du Xve siècle à aujourd'hui?

Daniel Leloup fait remarquer que les maisons de " bois et terrasses " apparaissent tardivement dans les textes, à partir du Xve siècle. Cette identification tardive doit-elle être mise en lien avec une existence tardive? Dès le xiII e siècle, Rennes se dote d'un bâtiment public en pan-de-bois : la Cohue. Faute de datations dendrochronologiques suffisantes, l'auteur se doit de rester évasif sur l'existence d'un habitat à pan-de-bois antérieur au Xve siècle. Plusieurs modèles cohabitent à la fin du Moyen Âge, ayant pour point commun une section importante des pièces de bois. D'un côté les maisons à fenestrage continu et poteaux élargis (3, rue du Chapitre; 